\title{
Long noncoding RNAs as novel predictors of survival in human cancer: a systematic review and meta-analysis
}

\author{
Stylianos Serghiou ${ }^{1,2}$, Aikaterini Kyriakopoulou ${ }^{3}$ and John P. A. Ioannidis $s^{4,5,6,7^{*}}$
}

\begin{abstract}
Background: Expression of various long noncoding RNAs (IncRNAs) may affect cancer prognosis. Here, we aim to gather and examine all evidence on the potential role of IncRNAs as novel predictors of survival in human cancer.

Methods: We systematically searched through PubMed, to identify all published studies reporting on the association between any individual IncRNA or group of IncRNAs with prognosis in human cancer (death or other clinical outcomes). Where appropriate, we then performed quantitative synthesis of those results using meta-analytic methods to identify the true effect size of IncRNAs on cancer prognosis. The reliability of those results was then examined using measures of heterogeneity and testing for selective reporting biases.

Results: Three hundred ninety-two studies were screened to eventually identify 111 eligible studies on 127 datasets. In total, these represented 16,754 independent participants pertaining to 53 individual and 6 grouped IncRNAs within a total of 19 cancer sites. Overall, $83 \%$ of the studies we identified addressed overall survival and 32 $\%$ of the studies addressed recurrence-free survival. For overall survival, $96 \%$ (88/92) of studies identified a statistically significant association of IncRNA expression to prognosis. Meta-analysis of 6 out of 7 IncRNAs for which three or more studies were available, identified statistically significant associations with overall survival. The IncRNA HOTAIR was by far the most broadly studied IncRNA ( $n=29$; of 111 studies) and featured a summary hazard ratio (HR) of 2.22 (95\% confidence interval (Cl), 1.86-2.65) with modest heterogeneity $\left(\mathrm{I}^{2}=49 \% ; 95 \% \mathrm{Cl}, 14-79 \%\right.$ ). Prominent excess significance was demonstrated across all meta-analyses ( $p$-value $=0.0003$ ), raising the possibility of substantial selective reporting biases.
\end{abstract}

Conclusions: Multiple IncRNAs have been shown to be strongly associated with prognosis in diverse cancers, but substantial bias cannot be excluded in this field and larger studies are needed to understand whether these prognostic information may eventually be useful.

Keywords: LncRNA, Cancer, Cancer biomarkers, Prognosis, Survival analysis, Excess significance, Small-study effects, Selective reporting biases

\section{Background}

Non-coding RNAs (ncRNAs) have been proposed in the last decade as regulators of cancer pathways and biomarkers of cancer outcomes [1-4]. Potentially informative biomarkers based on ncRNAs include microRNAs (miRs) [5] and the larger long non-coding RNAs

\footnotetext{
*Correspondence: jioannid@stanford.edu

${ }^{4}$ Stanford Prevention Research Center, Department of Medicine, Stanford

University School of Medicine Stanford, Stanford, CA 94305, USA

${ }^{5}$ Department of Health Research and Policy, Stanford University School of

Medicine, Stanford, CA 94305, USA

Full list of author information is available at the end of the article
}

(lncRNAs). NcRNAs were up to recently disregarded as 'junk' and despite constituting the large majority of RNAs being transcribed, their role in normal development and cellular physiology in health and disease is only recently becoming apparent $[2,6,7]$.

LncRNAs refer to any ncRNA consisting of more than 200 nucleotides. They are functionally heterogeneous molecules $[6,8]$, themselves sub-classified into large intergenic non-coding RNAs (lincRNA), transcribed ultraconserved regions (T-UCRs) and many others [2]. Of an estimated putative 140,000 different ncRNAs in 
total [9], lncRNAs are estimated to constitute proportionally the largest class, with the most comprehensive approach to date confirming 58,648 expressed lncRNAs [10]. Even though the function of lncRNAs is still being debated [11], certain lncRNAs have been implicated in functions related to regulation of gene expression in health and disease [2, 6-8, 12-15]. Well-studied examples include the lncRNA Xist, which initiates X-chromosome inactivation in female cells by recruiting repressive complexes to the Xchromosome under inactivation [16-18] and H19, which has been shown to play a significant role in genomic imprinting $[19,20]$.

Of particular interest however is, that it is now clear that lncRNAs are major players in tumorigenesis [7-9, 21-23]. In this context, the most well studied lncRNA is HOTAIR (HOmeobox (HOX) Transcript AntIsense RNA), which has been shown to recruit the PRC2 (Polycomb Repressive Complex 2) complex and eventually lead to epigenetic silencing of metastasis suppressor genes $[2,24]$.

More than 20 meta-analyses studying the role of IncRNAs in cancer prognosis have been published so far, all within the past 2 years. All of these studied a single lncRNA, either in relation to a specific cancer or to any cancer. The two most studied lncRNAs are MALAT1 and HOTAIR, which have been the subject of 10 and 7 meta-analyses respectively. The latest metaanalysis on MALAT1 for all cancer types showed that its upregulation is statistically significantly associated with poor overall survival (pooled hazard ratio [HR], 2.14; $95 \% \mathrm{CI}, 1.74-2.64$ ) with low between-study heterogeneity $\left(\mathrm{I}^{2}, 4.3 \%\right.$; $p$-value $\left.=0.399\right)$, on the basis of 9 studies [25]. The results were similar to the latest metaanalysis of HOTAIR (HR, 2.33; 95 \% CI, 1.77-3.09), but with significant between-study heterogeneity (Cochran's Q-test $p$-value $=0.016$ ), on the basis of 16 studies [26]. Interestingly, all meta-analyses published so far have been produced by Chinese groups and all identified a statistically significant association of all lncRNAs studied to prognosis in cancer. However, no systematic review and meta-analysis to-date has identified all lncRNAs studied in the context of cancer and to what extent these might be of prognostic significance.

In this paper, we aimed to examine the potential role of all lncRNAs ever investigated in the context of cancer survival prediction, as novel predictors of survival in human cancer. We utilized a field-wide metaanalysis approach [27] to systematically identify and examine all published papers trying to associate lncRNAs to prognosis in human cancer, and to quantitatively synthesize data directly related to prognosis wherever three or more studies on an IncRNA had been done.

\section{Methods}

\section{Systematic review}

This report has been structured on the basis of PRISMA [28].

\section{Eligibility criteria}

We considered published reports of a prospective or retrospective study design that had explored the association of any single or combination of stated lncRNAs to any of the following types of survival analysis: diseasespecific survival (DSS, duration of time from the day of diagnosis to the day of death due to cancer); metastasisfree survival (MFS, duration of time from day of diagnosis to the day of diagnosing a metastatic event); overall/ cumulative survival (OS, duration of time from day of diagnosis to the day of death due to any cause); progression/event/disease-free survival (PFS, duration of time from day of first treatment to the day evidence of cancer progression are identified or the patient dies of any cause); and recurrence-free survival (RFS, duration of time from day of cure from cancer to the day evidence of cancer progression/recurrence is identified). Survival analyses measuring different types of survival were treated separately at all times. Studies describing the association of individual or groups of IncRNAs with clinicopathologic variables (e.g. Stage, Grade, Distant metastasis, etc.), without specifically examining associations to any of the aforementioned survival analyses, were excluded. We likewise excluded cross-sectional studies and studies concerning genetic alterations of an lncRNA (e.g. polymorphisms or methylation patterns). Any kind of quantitative lncRNA analysis (quantitative real timePCR, in situ hybridization) was eligible.

For meta-analysis eligibility, a study had to also provide the effect size and confidence interval for the association of an individual or group of lncRNAs with any of the above survival outcomes, or report information through which this effect size and confidence interval could be calculated $[29,30]$. Wherever the same cohort had published more than one overlapping analysis, we only used the most encompassing data (for example, the classification of glioma would be preferred over glioblastoma multiforme). Two reviewers (S. Serghiou and A. Kyriakopoulou) identified eligible studies, and any contested articles were adjudicated by a third reviewer (J. P. A. Ioannidis).

\section{Information sources}

We systematically searched PubMed (1950 to September, 2015) for studies of any language that analyzed associations between lncRNAs and prognosis in human cancer. Our search strategy was developed in consideration of previous recommendations [30] and used the clinical queries prognosis filter, which has been reported to have 
an average estimated sensitivity of $92 \%$ for detecting articles related to prognosis [5, 31]. Our search term was: (Prognosis/Broad [filter]) AND ((lncRNA OR "Inc RNA" OR "long noncoding ribonucleic acid" OR "long noncoding RNA" OR "long non-coding ribonucleic acid" OR "long intergenic noncoding RNA" OR "long intergenic non-coding RNA" OR "long non-coding RNA" OR "long ncRNA" OR "lincRNA" OR "linc RNA") AND (cancer OR carcinoma OR tumor OR neoplas* OR tumour OR malignan* OR metastat* OR metastas* OR leukemia OR leukaemia OR lymphoma OR recurren* OR "lymph node" OR response) AND (Humans[Mesh] AND English[lang])). The search was last updated to include articles published through September 26, 2015.

\section{Study selection}

We used the programming language $\mathrm{R}$ [32] to remove duplicate records. Title and abstract were screened to identify relevant articles. The full manuscript of the relevant articles was screened against our eligibility criteria. Any uncertainties were resolved by consensus with JPA. Data were collected by two reviewers (SS, AK) and saved in a pre-designed extraction form on Google Sheets. Where information was ambiguous (such as, for example, mentioning multiple types of lncRNA quantification methods but not clarifying which one of those was used to provide the quantities utilized in the survival analysis), this was labelled as 'unclear'. An attempt was made to contact the authors when information was clearly logically inconsistent, as in for example quoting a hazard ratio (HR) outside the confidence interval (CI), but none replied. In one paper, the IncRNA expression level [33] was subdivided into low versus medium versus high; for this paper we only extracted the comparison between low versus high expression levels. The following data were extracted for all articles following the CHARMS checklist [34]: title; authors; year of publication; journal of publication; groupings (i.e. whether IncRNAs were studied one by one or in groups); what IncRNAs were studied; whether an agnostic approach to identifying the studied lncRNAs was used (where an agnostic approach would be one assuming no prior knowledge regarding the choice of IncRNA to be studied); cancer site (e.g. brain) and cancer subtype (e.g. glioblastoma multiforme); whether a paper reported clinicopathologic data of its sample and which ones; whether an attempt of associating those clinicopathologic data to lncRNAs was made and for which ones; whether an attempt of associating clinicopathologic data to prognosis was made and for which ones; whether an attempt was made to explain the clinical outcomes using non-clinical studies (in vivo, in vitro); the types of survival analyses used (as above); type of study design (prospective cohort, retrospective cohort, unreported); means of lncRNA quantitative analysis (qRT-PCR, qPCR, in situ hybridization (ISH), other); and whether the paper tried to make any non-clinical associations of the identified lncRNAs to cancer in vitro. For eligible articles we further extracted: country and city of origin of the study cohort, period of sample recruitment, range of sample ages, mean/median age with confidence interval, the population type (general population, non-general population (e.g. veterans), unreported), stage of cancer upon initial patient presentation, sample size, means of tissue preservation (frozen, paraffinembedded, both, other), any and what preoperative treatment was given, the total number of lncRNAs studied, the type of metric the paper used to characterize their results (hazard ratio, relative risk, odds ratio, $p$-value), type of analysis (i.e. univariable or multivariable), lncRNA quantity cut-off and its unit (i.e. the threshold based on which lncRNA expression was deemed upregulated or downregulated by the study), the sample size of each comparison group, the minimum and maximum participant follow-up time, the number of censored participants throughout followup and whether this was explicitly stated or read off the Kaplan-Meier curves, the HR and its CI (provided or inferred, e.g. from $p$-values and HR point estimates), the $p$-value and whether this was statistically significant at $p<0.05$ and whether an attempt to validate the reported results was made, and if so, what type of validation method was used (internal or external). For eligibility for meta-analysis, enough information to extract or calculate the natural logarithm of the hazard ratio and its variance must have been provided.

Whenever multiple datasets were combined into a single dataset to study a specific lncRNA, we only extracted the summary $\mathrm{HR}$, rather than extracting the HR respective to each constitutive dataset. If multiple datasets were assessed within the same study without being combined into a single dataset, we extracted the HR respective to each dataset, as they represent separate estimates. Where both the log-rank and Breslow tests were reported, only the log-rank was extracted. No cohort was used more than once and effect sizes describing a broader class of cancer (e.g. glioma) were preferred over subclassifications of that (e.g. glioblastoma multiforme). Three studies reported effect sizes that were excluded from further consideration because the quoted HRs contradicted the text [35] or they were either outside the CI or could not have possibly led to the quoted CI $[36,37]$; this led to complete exclusion of two out of these three studies $[35,37]$. Our database can be freely accessed here: https://goo.gl/EjCDAp.

\section{Risk of bias in individual studies}

Risk of bias in individual studies was assessed on the basis of the framework of assessing internal validity of articles 
dealing with prognosis $[30,38]$ and recommendations regarding reporting of biomarker studies [39, 40].

\section{Meta-analysis Summary measures and synthesis of results}

We meta-analyzed data on lncRNAs for which three or more estimates of their effect on a specific survival outcome were available. Therefore, meta-analyses were only done for OS and RFS. Effect sizes for OS and RFS were meta-analyzed separately. Our principal summary measure was the summary HR. Standard errors were calculated using: ln (upper limit of $\mathrm{CI} /$ lower limit of $\mathrm{CI}) /(2 \times 1.96)$. Estimates were synthesized using a random-effects model and estimated using the restricted maximum-likelihood ratio method. As previously described [27], four meta-analyses were done for each of: (1) multivariable data, (2) univariable data, (3) multivariable data combined with univariable data whenever multivariable data were unavailable (preferentially multivariable) and (4) univariable data combined with multivariable data whenever univariable data were unavailable (preferentially univariable). Given the similarity between the estimates of all four types of meta-analysis and the importance of multivariable modelling in prognostic studies, this report only quotes the estimates of the 'preferentially multivariable' meta-analysis; the rest can be found in Additional file 1: Table S2. For each estimate we provide the effect size and $95 \%$ CI. Heterogeneity was analyzed using the $\mathrm{Q}$ and $\mathrm{I}^{2}$ statistics and the $95 \% \mathrm{CI}$ of $\mathrm{I}^{2}$ was also calculated $[41,42]$. These analyses were done using $\mathrm{R}$ and the package metafor 1.9-8 [43]. Data were combined for each type of IncRNA regardless of cancer type. Wherever an lncRNA had been analyzed three or more times for one or more specific cancer type, a post hoc subgroup analysis per cancer type was done for that lncRNA.

\section{Risk of bias across studies}

Risk of publication bias is a significant concern in prognostic studies [30]. We explored excess significance for factors reported by at least 3 studies [44]. Briefly, for every meta-analyzed risk factor we compare the number of observed significant results $(\mathrm{O})$ at $\alpha=0.05$, to the number of expected significant results $(E)$, where $E=$ sum of power of each study within a specific meta-analysis. Power was calculated taking as plausible effect for the risk factor the effect seen in the most precise study (lowest standard error). The difference between $\mathrm{O}$ and $\mathrm{E}$ was assessed using a twotailed binomial test, with $\alpha=0.1$, as previously suggested [45]. $\mathrm{O}$ and $\mathrm{E}$ were also summed and compared across all meta-analyses.

\section{Results}

Literature search and description of studies

We initially identified 397 records, from which 286 were excluded (Fig. 1), leaving us with 111 studies eligible for systematic review (Additional file 2), of which 85 were also eligible for meta-analysis. The 111 studies utilized 127 datasets to produce their analyses (four studies utilized two datasets, three studies utilized three datasets and two studies utilized four datasets). No new studies were imported through reference checking.

Of 127 identified datasets, only 2 were reported to represent a prospective cohort; of the rest, 19 were reported to represent a retrospective cohort and there were no relevant information for the remaining 106 datasets. No report specified what type of population their samples came from and for 113/127 datasets we have no information as to what sampling method was used to obtain the sample. For the remaining datasets, consecutive sampling was stated to have been used in 5 and random sampling in 4 datasets; 5 datasets were based on all patients ever seen by the clinic. Sampling method was disproportionately frequently reported for studies coming from the USA (4/9). A total of 94/127 datasets came from Asia (78 from China), followed by Europe (15/127) and America (13/127); there was no reported country of origin for 2 datasets and 3 datasets contained patients from multiple continents; the latter were multi-center cohorts. A total of 16,754 different patients were enrolled within these studies (avoiding double-counting samples that had been used for two or more analyses). Median sample size was 90 (IQR, 82; range, 30-997) and 69/127 datasets contained less than 100 participants (50 of which datasets came from China).

\section{Mapping of IncRNA prognostic data}

The eligible reports studied 18 types of cancer, top three most studied of which were gastric cancer $(n=16$ datasets), lung cancer $(n=15)$ and colorectal cancer $(n=15)$ (Table 1). Almost half of the reports studied cancer related to the gastrointestinal tract (57/127 datasets). OS was assessed in 92/111 studies (83 \%), RFS in 36 (32\%), DSS in 10 (9\%), MFS in $9(8 \%)$ and PFS in $6(5 \%)$. The majority of studies did not appear to choose what lncRNAs to study on the basis of agnostic reports (77 \%, $85 / 111)$. For $98 / 127$ datasets $(77 \%)$, there was no information regarding adjuvant treatment; for the 29 studies providing information regarding treatment, only 4 datasets indicated that their patients were treated homogeneously. In addition to survival analysis, $68 \%(76 / 111)$ of the identified studies attempted to further study their chosen IncRNAs in vitro, to corroborate the results of their survival analyses with mechanistic insights into the function of their chosen lncRNAs. Across 66 studies reporting multivariable analyses, 42 adjusted for stage of 

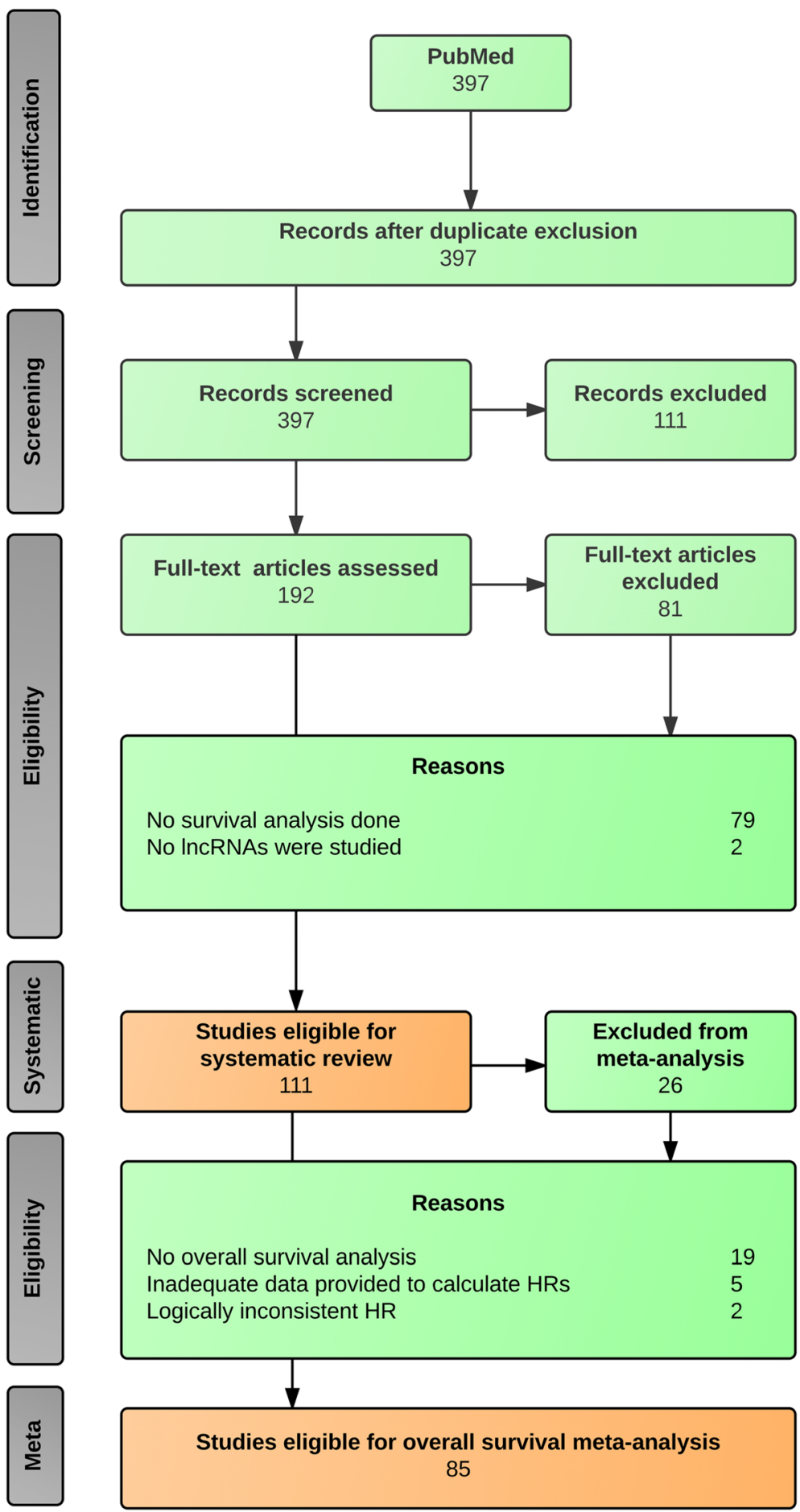

Fig. 1 A flow diagram demonstrating the study selection process. Out of 397 identified records, 111 were chosen for systematic review and 85 for overall survival meta-analysis. Duplicate exclusion refers to the process of asserting that each paper is only represented once within our set of records. Initially, 111 records with titles seemingly irrelevant to the objectives of our study were excluded, following which another 81 records were excluded after reading through the remaining 192 papers, for the reasons identified within the diagram. This process led to the identification of 111 papers eligible for systematic review. We then applied our meta-analysis eligibility criteria to these papers, on the basis of which 26 were excluded, for the reasons identified in the diagram. This process led to the identification of 85 papers eligible for meta-analysis 
Table 1 Descriptive statistics of eligible studies

\begin{tabular}{|c|c|c|}
\hline Characteristic & Subgroups & Frequency (\%) \\
\hline \multirow[t]{5}{*}{ Year } & 2000-2013 & $13(12 \%)$ \\
\hline & 2013-2014 & $23(21 \%)$ \\
\hline & 2014-2014 & $0(0 \%)$ \\
\hline & 2014-2015 & 75 (68 \%) \\
\hline & $\mathrm{N}$ & $111(100 \%)$ \\
\hline \multirow[t]{20}{*}{ Cancer site } & Gastric & $16(13 \%)$ \\
\hline & Colorectal & $15(12 \%)$ \\
\hline & Lung & $15(12 \%)$ \\
\hline & Brain & $12(9 \%)$ \\
\hline & Esophageal & 10 (8 \%) \\
\hline & Prostate & $10(8 \%)$ \\
\hline & Hepatic & $9(7 \%)$ \\
\hline & Breast & $6(5 \%)$ \\
\hline & Pancreatic & $6(5 \%)$ \\
\hline & Cervical & $5(4 \%)$ \\
\hline & Head and neck & $4(3 \%)$ \\
\hline & Ovarian & $4(3 \%)$ \\
\hline & Renal & $4(3 \%)$ \\
\hline & Urinary bladder & $4(3 \%)$ \\
\hline & Any & 2 (2 \%) \\
\hline & Hematologic & 2 (2 \%) \\
\hline & Endometrial & $1(1 \%)$ \\
\hline & GIST & $1(1 \%)$ \\
\hline & Neuroblastoma & $1(1 \%)$ \\
\hline & $\mathrm{N}$ & $127(100 \%)$ \\
\hline \multirow[t]{4}{*}{ Agnostic $^{a}$} & No & $85(77 \%)$ \\
\hline & Yes & $21(19 \%)$ \\
\hline & Agnostic parent & $5(5 \%)$ \\
\hline & $\mathrm{N}$ & $111(100 \%)$ \\
\hline \multirow[t]{6}{*}{ Survival analysis } & OS & $92(83 \%)$ \\
\hline & RFS & 36 (32 \%) \\
\hline & DSS & $10(9 \%)$ \\
\hline & MFS & $9(8 \%)$ \\
\hline & PFS & $6(5 \%)$ \\
\hline & $\mathrm{N}$ & $111(100 \%)$ \\
\hline \multirow[t]{7}{*}{ Quantification method } & qRT-PCR & $84(66 \%)$ \\
\hline & ISH & $28(22 \%)$ \\
\hline & qPCR & $11(9 \%)$ \\
\hline & qRT-PCR or ISH & $2(2 \%)$ \\
\hline & RT-qPCR & $1(1 \%)$ \\
\hline & Unreported & $1(1 \%)$ \\
\hline & $\mathrm{N}$ & $127(100 \%)$ \\
\hline \multirow[t]{2}{*}{ Continent } & Asia & 94 (74 \%) \\
\hline & Europe & 15 (12 \%) \\
\hline
\end{tabular}

Table 1 Descriptive statistics of eligible studies (Continued)

\begin{tabular}{|c|c|c|}
\hline & America & $13(10 \%)$ \\
\hline & Multiple & $3(2 \%)$ \\
\hline & Unreported & 2 (2 \%) \\
\hline & $\mathrm{N}$ & $127(100 \%)$ \\
\hline \multirow[t]{4}{*}{ Study design } & Unreported & 106 (83\%) \\
\hline & Retrospective & $19(15 \%)$ \\
\hline & Prospective & $2(2 \%)$ \\
\hline & $\mathrm{N}$ & $127(100 \%)$ \\
\hline \multirow[t]{5}{*}{ Sampling method } & Unreported & $113(89 \%)$ \\
\hline & Consecutive & $5(4 \%)$ \\
\hline & Population & $5(4 \%)$ \\
\hline & Random & $4(3 \%)$ \\
\hline & $\mathrm{N}$ & $127(100 \%)$ \\
\hline \multirow[t]{6}{*}{ Tissue preservation ${ }^{b}$} & L & 66 (52 \%) \\
\hline & Unreported & $34(27 \%)$ \\
\hline & $P$ & $18(14 \%)$ \\
\hline & $L P$ & $6(5 \%)$ \\
\hline & L + RNALater & $3(2 \%)$ \\
\hline & $\mathrm{N}$ & $127(100 \%)$ \\
\hline \multirow[t]{4}{*}{ Pre-biopsy treatment } & No & 77 (61%) \\
\hline & Unreported & $46(36 \%)$ \\
\hline & Yes & $4(3 \%)$ \\
\hline & $\mathrm{N}$ & $127(100 \%)$ \\
\hline \multirow[t]{4}{*}{ Post-biopsy treatment } & Unreported & $98(77 \%)$ \\
\hline & Yes & $27(21 \%)$ \\
\hline & No & $2(2 \%)$ \\
\hline & $\mathrm{N}$ & $127(100 \%)$ \\
\hline \multirow{5}{*}{$\begin{array}{l}\text { Total number of IncRNAs } \\
\text { studied }\end{array}$} & 1 & 87 (78 \%) \\
\hline & $2-10$ & $4(4 \%)$ \\
\hline & $11-45033$ & $15(14 \%)$ \\
\hline & Unreported & $5(5 \%)$ \\
\hline & $\mathrm{N}$ & $111(100 \%)$ \\
\hline \multirow[t]{3}{*}{ Outcomes } & Clinical and Non-clinical & $76(68 \%)$ \\
\hline & Clinical only & 35 (32 \%) \\
\hline & $\mathrm{N}$ & $111(100 \%)$ \\
\hline \multirow{6}{*}{$\begin{array}{l}\text { Use of validation method } \\
\text { for survival }\end{array}$} & Unreported & $99(89 \%)$ \\
\hline & External & $5(5 \%)$ \\
\hline & Internal & $4(4 \%)$ \\
\hline & Both & $2(2 \%)$ \\
\hline & Yes & $1(1 \%)$ \\
\hline & $\mathrm{N}$ & $111(100 \%)$ \\
\hline
\end{tabular}

These data are based on 111 studies of 127 datasets. $\mathrm{N}$ refers to the total number of observations for each characteristic

${ }^{a}$ Agnostic studies are those in which no prior knowledge is assumed regarding the choice of IncRNA to be studied

b Tissue preservation: L (liquid nitrogen), P (paraffin-embedded), LP (liquid nitrogen and/or paraffin-embedded 


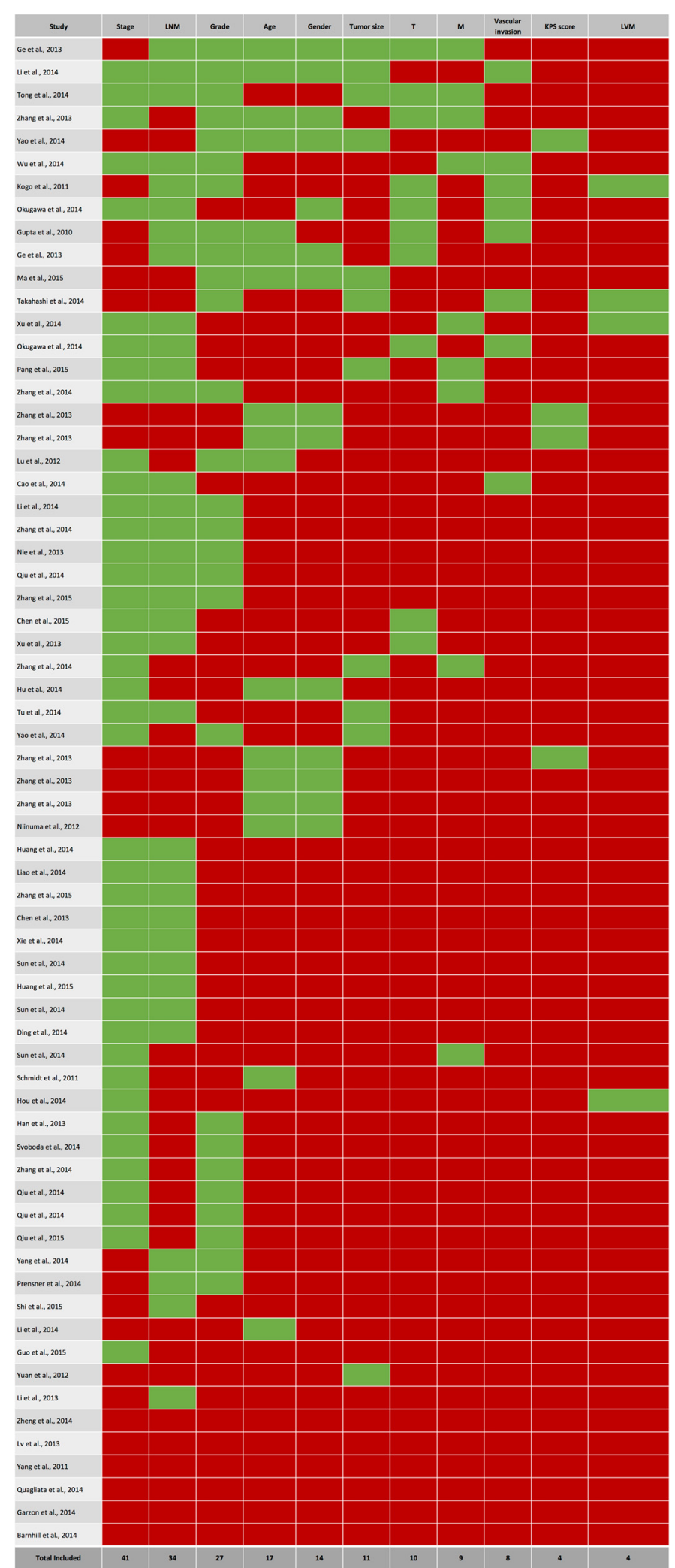

Fig. 2 (See legend on next page.) 
(See figure on previous page.)

Fig. 2 The covariates used within the multivariable models fitted by each paper. This is a data microarray in which the studies run along the $\mathrm{Y}$-axis and the covariates run along the X-axis. Only the factors used three or more times are shown in this figure for convenience; refer to Additional file 3: Figure S1 for a data microarray illustrating all covariates studied. Rows and columns are ordered in descending order, based on how many times each covariate was included in the multivariable models fitted by each study. Where patterns were similar between studies or covariates, those papers or covariates were placed next to each other. It is evident that very few studies included the same covariates within their models and that less than half of the studies included both Stage and Grade within those models. Interestingly, according to Additional file 3: Figure S1, the majority of studies included at least one covariate within their model that had not been included in any other study. Green = Included in the multivariable model; Red = Not included in the multivariable model. LNM = Lymph node metastasis; $T=$ Depth of invasion; $M=$ Metastasis; KPS score $=$ Karnofsky Performance Status score (a measure of functional impairment); LVM = Lymphovascular metastasis

cancer (or all three components of the TNM staging) and 27 for grade of cancer; only 19/66 studies adjusted for both. Figure 2 displays a microarray of the covariates that have been studied more than three times within multivariable analyses (Additional file 3: Figure S1 displays the complete data microarray). Out of all 66 studies, $20(30 \%)$ studies adjusted for the same factors as at least one other paper and the most commonly encountered combination of factors adjusted for was Stage and Lymph Node Metastasis, which was seen in 6/66 studies. The median number of adjustment combinations matching between at least two papers was 1 (IQR, 0).

\section{Overall survival}

Out of 92 studies reporting on OS, 87 studies (representing 111/127 analyses, as explained in Additional file 4: Table S1) provided effect estimates, out of which two were completely excluded due to reporting inconsistent effect sizes, as indicated in the Methods [35, 37]. The 85 remaining studies provided effect estimates on 53 lncRNAs and 6 multi-lncRNA risk score scales. The three most frequently studied lncRNAs within OS analyses were HOTAIR ( $n=29$ effect estimates), MALAT1 $(n=8)$ and GAS 5, H19 and PVT1 $(n=4$ for each). Most individual lncRNAs (42/53) were only studied once (Table 2). Only 7 lncRNAs were studied at least three times in association to OS and for 6 of them more than half of the studies showed statistically significant $p$-values. These lncRNAs were studied in the context of a median of 4 different types of cancer (IQR, 3). Out of the 52 individual or groups of lncRNAs studied less than three times, 44 were always reported significantly associated to OS. Overall, of the 92 studies reporting on OS (but not necessarily quoting an effect estimate), 88 (96\%) reported at least one statistically significant result for association with prognosis.

\section{Meta-analysis for overall survival}

A meta-analysis of OS was done for all 7 individual or groups of lncRNAs having been studied three or more times (Fig. 3; Table 3; Additional file 1: Table S2). For $p$-value $<0.0005,5$ lncRNAs were statistically significantly associated to OS in all of our meta-analyses
Table 2 Details of the IncRNAs studied

\begin{tabular}{|c|c|c|c|c|}
\hline LncRNA & $\begin{array}{l}\text { Times } \\
\text { studied }\end{array}$ & $\begin{array}{l}\text { Number of } \\
\text { cancer types } \\
\text { (sample size) }\end{array}$ & $\begin{array}{l}\text { Median } \\
\text { (IQR) }\end{array}$ & $\begin{array}{l}\text { Times } \\
\text { significant (\%) }\end{array}$ \\
\hline HOTAIR & 29 & $13(3886)$ & $100(69)$ & 28 (97 \%) \\
\hline MALAT1 & 8 & $7(1135)$ & $136(53)$ & $5(62 \%)$ \\
\hline $\mathrm{H} 19$ & 4 & $2(440)$ & $77(57)$ & $2(50 \%)$ \\
\hline PVT1 & 4 & $4(420)$ & $87(24)$ & $4(100 \%)$ \\
\hline GAS5 & 4 & $4(369)$ & $96(19)$ & $4(100 \%)$ \\
\hline SChLAP1 & 3 & 1 (1396) & $357(440)$ & $3(100 \%)$ \\
\hline $\begin{array}{l}6 \text { IncRNA risk } \\
\text { score }\end{array}$ & 3 & $1(281)$ & $42(94)$ & 1 (33\%) \\
\hline CCAT2 & 2 & $2(1226)$ & $613(384)$ & 1 (50 \%) \\
\hline $\begin{array}{l}\text { LncR1 vs LncR2 vs } \\
\text { LncR3 }\end{array}$ & 2 & $1(759)$ & $380(96)$ & $2(100 \%)$ \\
\hline ENSG00000261582 & 2 & $2(576)$ & 288 (199) & $2(100 \%)$ \\
\hline $\mathrm{MVIH}$ & 2 & $2(257)$ & $128(86)$ & 2 (100 \%) \\
\hline LOC285194 & 2 & $2(227)$ & $114(28)$ & $2(100 \%)$ \\
\hline PCAT1 & 2 & $2(212)$ & $106(2)$ & 2 (100\%) \\
\hline SPRY4-IT1 & 2 & $2(190)$ & $95(3)$ & $2(100 \%)$ \\
\hline UCA1 & 2 & $2(170)$ & $85(5)$ & 2 (100 \%) \\
\hline GHET1 & 2 & $2(122)$ & $61(19)$ & $2(100 \%)$ \\
\hline MEG3 & 2 & $2(116)$ & $58(14)$ & 2 (100\%) \\
\hline
\end{tabular}

The following IncRNAs were studied once and found statistically significant: LINC00968, LINC01234, LINC00476, FLG-AS1, HOTTIP, TC0101686, TC0100223. The following IncRNAs were studied once and were not found significant: lincUBC1, KIAA0495, PART1, MGC21881, MIAT, PAR5, ADAMTS9-AS2, BCAR4,

XLOC_010588, FOXCUT, 3 InCRNA risk score, FENDRR, HIF1A-AS2, ANRIL, GAPLINC, MRUL, HEIH, HOXA13, 48 InCRNA risk score, BANCR, ZXF1, CARLo-5, GAS6-AS1, Sox2ot, TUG1, NAG7 - LINC00312, CAI2, TC0101441, ENST00000480739, BC008363, 80-gene SChLAP1 signature risk score, 167-gene SChLAP1 signature risk score, CADM1-AS1, RCCRT1, CCAT1. Significance in the table refers to $p$-value $<0.05$, as this is what had been used by these studies

The 'Times studied' column refers to how many studies investigated each IncRNA. The 'Number of cancer types' column indicates in how many different cancer types each IncRNA was studied, with the total number of participants used to study each IncRNA in brackets. The 'Median' column indicates the median sample size for each cohort used to study each IncRNA, with the interquartile range (IQR) in brackets. The last column indicates how many times each IncRNA was found to be statistically significantly associated to prognosis and in brackets the relation of how many times it was found to be significant versus how many times it was studied 


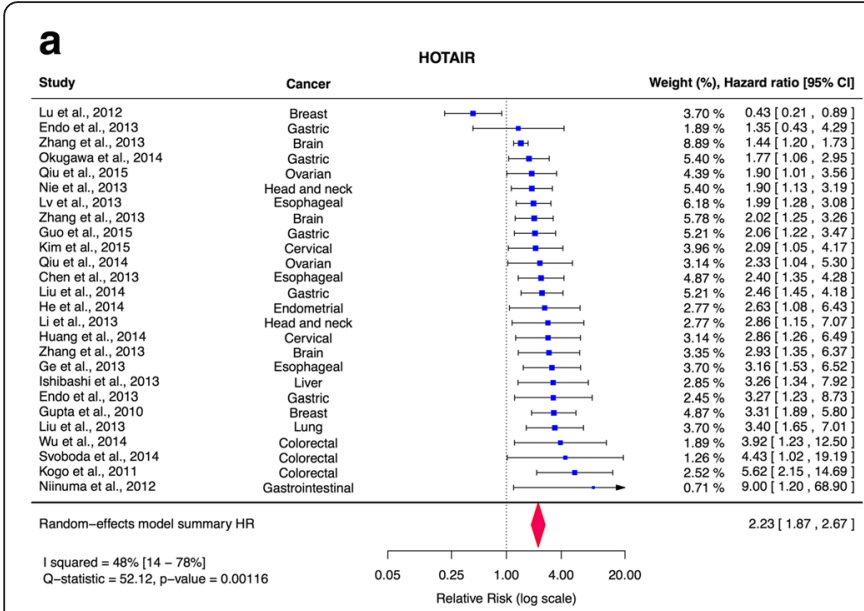

\section{d}

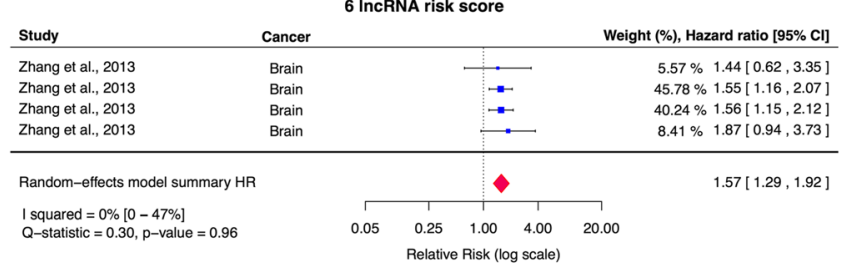

\section{f}

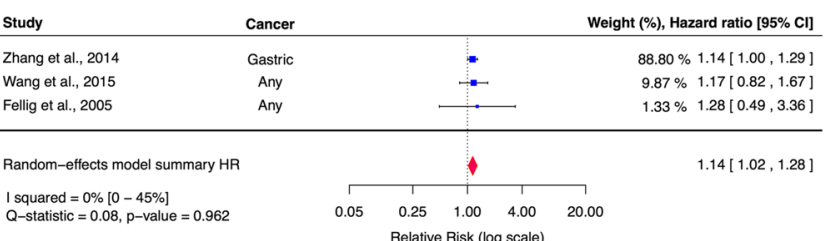

b

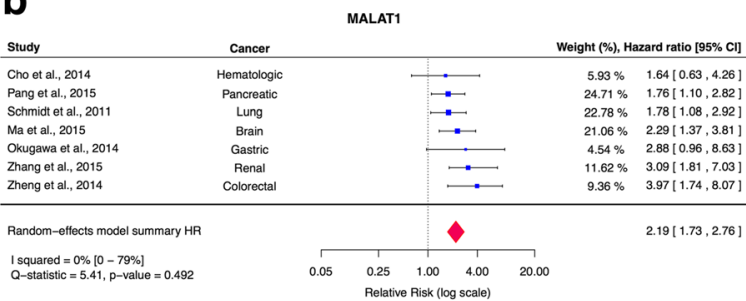

C

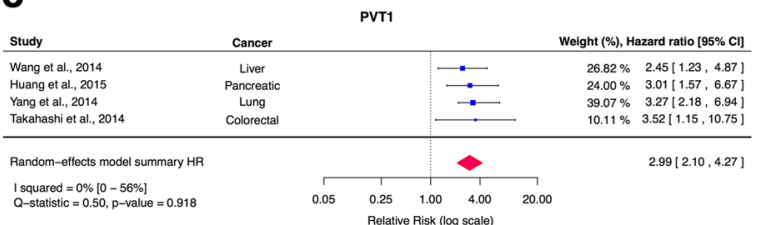

e

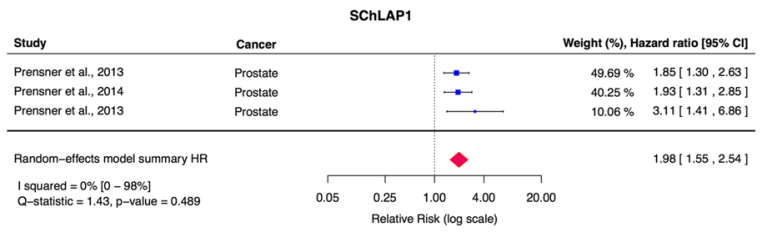

g

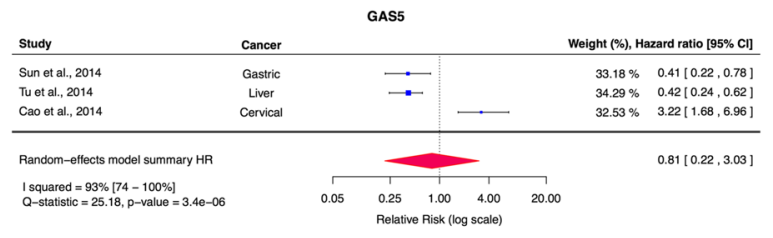

Fig. 3 Forest plots for OS meta-analyses. We hereby illustrate the IncRNAs for which three or more studies reported OS. Each panel a-g corresponds to the meta-analysis of a different IncRNA: (a) HOTAIR, (b) MALAT1, (c) PVT1, (d) 6 IncRNA risk score, (e) SChLAP1, (f) H19 and (g) GAS5. The effect size for the estimate of each study is presented as a blue square proportional in size to the weight of that study. The confidence interval around that effect size is presented as a horizontal line. Where the confidence interval exceeds the range of our plot, an arrow has been placed. The vertical line across these estimates represents $\mathrm{HR}=1$ and any horizontal line crossing this vertical line represents a non-statistically significant result. The summary effect size is presented as a rhombus, the center of which represents the summary effect size and the width of which represents its confidence interval. It is evident that almost all studies quoted statistically significant results and that according to the available data, all meta-analyzed IncRNAs, apart from GAS5 (panel g), are statistically significantly associated to prognosis of OS in cancer. However, high between-study heterogeneity (based on the range of $\mathrm{I}^{2}$ estimates) indicates that these summary effect sizes are unreliable

Table 3 The results of our meta-analysis for each IncRNA using 'primarily multivariable' data

\begin{tabular}{llllll}
\hline LncRNA & Studies & HR $(95 \% \mathrm{Cl})$ & $P$-value & $\mathrm{I}^{2}(95 \% \mathrm{Cl})$ & Observed (Expected, $p$-value $)$ \\
\hline HOTAIR & 26 & $2.22(1.86-2.65)$ & 0.0000 & $49 \%(14-79 \%)$ & $25(18.2, p$-value $=0.002)$ \\
MALAT1 & 7 & $2.03(1.64-2.52)$ & 0.0000 & $0 \%(0-85 \%)$ & $5(4.1, p$-value $=0.707)$ \\
6 IncRNA risk score & 4 & $1.57(1.29-1.92)$ & 0.0000 & $0 \%(0-47 \%)$ & $2(2.1, p$-value $=1.000)$ \\
GAS5 & 4 & $0.81(0.33-2.00)$ & 0.6479 & $94 \%(80-100 \%)$ & $4(2.1, p$-value $=0.128)$ \\
H19 & 4 & $1.16(1.04-1.29)$ & 0.0100 & $0 \%(0-98 \%)$ & $2(0.8, p$-value $=0.170)$ \\
PVT1 & 4 & $2.99(2.10-4.27)$ & 0.0000 & $0 \%(0-56 \%)$ & $4(2.7, p$-value $=0.309)$ \\
SChLAP1 & 3 & $1.98(1.55-2.54)$ & 0.0000 & $0 \%(0-98 \%)$ & $3(2.9, p$-value $=1.000)$ \\
\hline
\end{tabular}

'Studies' refers to the number of studies included in the meta-analysis of each IncRNA. HR = Hazard Ratio, $95 \% \mathrm{Cl}=95 \%$ Confidence Interval. ${ }^{2}$ is a measure of between-study heterogeneity. The last column refers to how many statistically significant results had been reported by the included studies (Observed, $O)$, how many were expected to be reported on the basis of each study's power (Expected, E) and whether $\mathrm{O}$ and $\mathrm{E}$ are statistically significantly different from each other for each meta-analysis ( $p$-value). Please refer to Additional file 1: Table S2 for a table illustrating all meta-analyses done (not only the one for primarily multivariable data) with all of the measures calculated 
(HOTAIR, MALAT1, 6 lncRNA risk score, PVT1, SChLAP1) and 6/7 were statistically significant in all of our meta-analyses at $p$-value $<0.05$ (H19; Additional file 1: Table S2). An increase in cellular expression of these lncRNAs was statistically significantly associated to a decrease in overall survival; GAS5 was not statistically significantly associated to $O S$ in our metaanalyses. The funnel plot for HOTAIR (Fig. 4), which is the only lncRNA studied 10 or more times, indicates significant small-study effects $(p$-value $=0.0006)$, and this may be suggestive of publication bias. The summary effect size for HOTAIR also displays a moderate amount of between-study heterogeneity ( $\mathrm{I}^{2}, 48 \%$; $95 \%$ CI, 14-78\%). The summary effects for the effect of HOTAIR on OS in cancers for which it was studied 3 or more times were: colorectal cancer (HR, 4.76; $95 \%$ CI, 2.46-9.21), esophageal cancer (HR, 2.29; 95 \% CI, 1.68-3.12) and glioma (HR, 1.71; 95 \% CI, 1.25-2.34).

\section{Other meta-analyses}

The only type of survival analysis other than OS studied 3 or more times in relation to a specific lncRNA was MFS for HOTAIR. This was investigated within 4 different studies in relation to 4 different cancers (breast, colorectal, esophageal, head and neck). Meta-analysis of these studies identified a summary HR of $2.54(95 \% \mathrm{CI}$, $1.62-3.98)$ with no statistically significant heterogeneity (Q-statistic, 5.16; $p$-value $=0.16$ ).

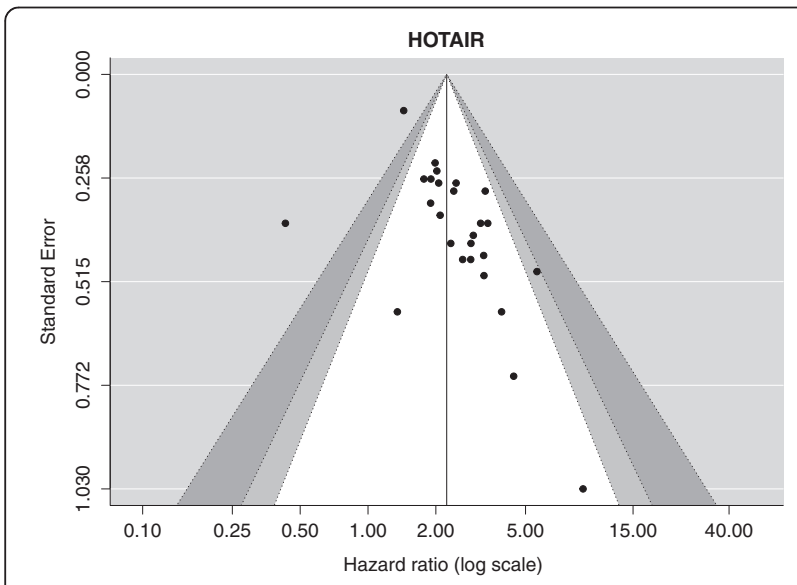

Fig. 4 Funnel plot for the OS meta-analysis of HOTAIR. The meta-analysis for HOTAIR was analyzed with a funnel plot because it exceeded the pre-requisite of 10 studies. The $\mathrm{Y}$-axis represents the Standard Error (SE), which serves as a measure of precision, where the higher the SE, the less precise the study. The HR has been plotted along the X-axis. The black dots map the effect size of HOTAIR on OS as this has been identified by each study. The light grey and dark grey areas respectively denote the $95 \%$ and $99 \% \mathrm{Cl}$ around the summary effect size. According to this plot, it is clear that the least precise studies tend to overestimate the effect size of HOTAIR on OS, skewing the summary effect size to the right (i.e. leading to a more strongly positive summary effect size)

\section{Heterogeneity metrics and excess significance}

Statistically significant heterogeneity was only observed in HOTAIR analyses, but substantial estimates of $\mathrm{I}^{2}$ were common. For HOTAIR and OS, a sensitivity analysis excluding the only study reporting an inverse correlation of HOTAIR to cancer survival [33] generated a HR of 2.30 (95 \% CI, 1.97-2.70) with $\mathrm{I}^{2}=0$ \% (95 \% CI, 0-59 \%); for all other meta-analyses, no single study produced a major change in the $\mathrm{I}^{2}$.

There was excess significance across the whole field for overall survival and the binomial distribution revealed a two-tailed $p$-value of 0.0003 , with $\mathrm{O}=42$ statistically significant results and $\mathrm{E}=30$ expected statistically significant results across all meta-analyses with 3 or more studies each on OS. As far as excess significance within lncRNAs studied 5 or more times is concerned, there was significant excess significance documented for HOTAIR ( $p$-value $=0.002)$, but not MALAT1 $(p$-value $=0.46$ ).

\section{Discussion}

In this systematic review and meta-analysis we have tried to gather all published papers evaluating the prognostic ability of IncRNAs in cancer. We have identified that a large number of lncRNAs have been evaluated within the context of cancer prognosis. Most of them have been evaluated only once in a published paper. Almost all of the published papers report that lncRNAs are statistically significant predictors of survival. There was often substantial heterogeneity between studies in the strength of the predictive effect. There was also strong evidence for small-study effects and for excess significance. This picture may be due to genuine differences across studies, such as different cancers and populations under study, and different adjustments made in multivariable models. However, it is also highly compatible with the presence of substantial publication bias and other selective reporting bias in this field resulting in exaggerated effects in mostly small studies (most of which coming from China) and in an implausibly high prevalence of nominally significant results.

It is well recognized that published literature on prognosis and the identification of prognostic markers is characterized by poor methodological quality, significant publication bias and wide heterogeneity in aspects of sample selection, such as pre/post-biopsy treatment or tissue preservation methods, and analysis, such as multivariable modelling and determination of cutoff values $[30,46]$. As such, meta-analyses of prognostic studies may elicit summary effect sizes that are unrealistic [47]. An evaluation of studies investigating the association of TP53 to risk of death by head and neck squamous cell carcinoma, identified that even though readily available effect sizes would confirm that TP53 is a strongly 
significant prognostic factor, after standardizing definitions of TP53 status and outcomes across papers and retrieving non-readily available information, this association was completely abrogated [48]. These issues may also apply to the lncRNA literature. No two studies of our dataset were identical in all of IncRNA, cancer site, cut-off value and multivariable modelling, suggesting substantial room for selective reporting of analyses that could be done with very different models and definitions. Moreover, we suspect that publication bias may also be operating in the field.

Of particular interest is the excess significance we identified across the field ( $p$-value $=0.0003$ ). Despite the poor translation of cancer biomarkers into clinical practice [39, 49-51], out of 1575 studies on cancer biomarkers published in 2005, $95.8 \%$ reported statistically significant results and only $1.3 \%$ did not report any kind of statistically significant results [52]. Indeed, as we have shown, this pattern is also prominent in the lncRNA cancer prognosis literature.

One way of reducing the selective reporting biases that have led to the above status quo and thus reducing lack of translatability, is transparency. The need to improve transparency has been mentioned repeatedly [39, 53]. Guidelines have been proposed to improve the reporting of prognostic markers (REMARK) [39, 51], multivariable prediction models (TRIPOD) [54] and genetic risk prediction studies [55]. Wider adoption of these guidelines may increase transparency, but it is unknown whether it will suffice to markedly reduce selective reporting.

In our cohort of studies, the extent of unreported items in Table 1, did not inspire confidence in transparency and completeness of reporting practices. We also documented minimal use of validation (12/111 studies, $11 \%)$, despite reports stressing the necessity and importance of validation in identifying true effect size for prognostic tools [56, 57]. Furthermore, more than half of the identified studies had a sample size of less than 100 . Small studies are known, both theoretically and empirically, to be associated with inflated estimates of effect size [58], not as much due to their limited sample size, as for lower quality standards, publication bias and selective reporting [59], which is why they lead to so-called 'small-study effects'. Even though these have mostly been studied within the context of randomized-controlled trials, where they have been associated with a larger average effect size and at least double the between-study heterogeneity found in larger studies [60], similar problems may occur also in prognostic study research [43]. The meta-analysis for HOTAIR, which is the most widely studied lncRNA in the context of cancer prognosis, clearly indicates that smaller studies tend to be less precise and report a higher effect size than larger studies. Inflated effects are common in biomarker studies [61], and this may apply also for the results of lncRNAs.
Another interesting point of note is the Chinese provenance of most papers in our collection of eligible studies $(78 / 111,70 \%)$. In a previous analysis of genetic studies, it was shown that there is a vast Chinese literature, and that papers from China tend to utilize smaller sample sizes yet reach statistical significance far more commonly than other papers [62]. This was attributed to more prominent publication bias against null results or other kinds of selection bias in pursuit of statistically significant results. Discrepancies between the Chinese literature and the rest of the world were also found in published meta-analyses of genomic data [63]. Chinese meta-analyses (1) focused on the results of studies investigating individual candidate genes rather than the results of genome-wide association studies and (2) used nominal significance (i.e. $p$-value $<0.05$ ) rather than genome-wide $p$-value thresholds to identify statistically significant results.

Although there has been an explosion in the amount of identified potential biomarkers due to high throughput methods, unlike traditional methods of identifying molecules directly relevant to a known cellular event [49], very few have made their way to clinical practice, due to lack of appropriate evidence [50, 64, 65]. An important aspect in ascribing usefulness to a novel biomarker is their ability to add further predictive value, over and above the one already possible using known prognostic factors. Unfortunately, in our sample, despite most multivariable analyses identifying lncRNAs as a statistically significant predictor, only about $30 \%$ of the reported prognostic effects were adjusted for the two classically most relevant predictors of cancer prognosis (i.e. Stage and Grade).

\section{Limitations}

Our analysis has several limitations. First, given that this report is only based on the results of a single database (PubMed), it is possible that relevant papers may have been missed. Second, our analysis utilized the Medical Subject Heading (MeSH) 'Humans' to limit our search results to those studies conducted in humans. Even though this is accepted practice and has been used previously in similar studies [5], that label is added to papers at the point of indexing, and thus some papers that were published close to our search date (September 26, 2015) and had not been MeSH-labeled yet, would have been missed. We performed an updated search (June 5, 2016) for papers that did not have a Human [MeSH] and had been published before 2015 and found only two small studies $[66,67]$ that could potentially qualify for inclusion for the outcome of survival. This is a field with prolific literature and a substantial number of papers have continued to appear after our September 2015 search and will probably continue to appear in the near future. Third, our meta-analysis has attempted to combine 
multiple studies that are known to be heterogeneous in terms of cancer site and provenance of patient populations. Our estimates of heterogeneity metrics have wide $95 \%$ confidence intervals [42]. Fourth, on 51 occasions we had to calculate HRs ourselves based on data provided within the papers, which may not have provided the most accurate estimate of the HR possible, as most of the time these data were extracted from Kaplan-Meier curves. However, this practice has not been shown to yield results significantly different from direct methods of HR estimation [29]. Fifth, even though every effort was made to exclude analyses of the same lncRNA using the same dataset of patients, it is possible that some overlapping data have been included, if their authors have made no hint as to the presence of overlap.

\section{Conclusions}

In conclusion, we have gathered a substantial amount of prognostic data regarding the association of various lncRNAs and survival. Our analysis identified a significant number of studies, most of which have been published within the last 2 years and most of which are of small sample size. Even though our systematic review and meta-analyses identified that almost all lncRNAs identified are statistically significant predictors of OS, it is very difficult to know the importance of these associations, given the detection of excess significance, smallstudy effects and the known difficulties with analyzing prognostic studies. Larger studies, ideally with collaborative teams using standardized approaches to measurement, adjustment, analysis, and reporting, will offer better insights into the prognostic value of lncRNAs.

\section{Additional files}

Additional file 1: Table S2. A table presenting all meta-analyses done. 'Analysis' refers to the the type of data used in each metaanalysis, as this was explained in Methods. 'Studies' refers to the number of studies included within each meta-analysis. Columns 'HR' and ' $95 \% \mathrm{Cl}$ ' refer to the summary Hazard Ratio (HR) of each metaanalysis with its $95 \%$ Confidence Interval (CI) (lower and upper limit). 'Tau' refers to the squared root of the estimate of between-study variance in each of our random-effects meta-analyses. Columns 'I and ' $95 \% \mathrm{Cl}$ ' refer to a measure of between-study heterogeneity and its corresponding $95 \% \mathrm{Cl}$. 'Q-statistic' and its 'P-value' refer to Cochran's $\mathrm{Q}$ measure of heterogeneity with its $p$-value. 'Observed', 'Expected' and 'P-value (binomial)' refer to the observed and expected amount of statistically significant results and the comparison between the two, as this was described in Methods. (XLSX $50.7 \mathrm{~kb}$ )

Additional file 2: The studies eligible for systematic review. (DOC $444 \mathrm{~kb}$ )

Additional file 3: Figure S1. The covariates included within the multivariable models fitted by each paper. This is a data microarray in which the studies run along the $Y$-axis and the covariates run along the $X$-axis. Rows and columns are ordered in descending order, based on the total number each covariate was included in the multivariable models fitted by each study. Where patterns were similar between studies or covariates, those studies or covariates were placed next to each other. (PDF $82 \mathrm{~kb})$
Additional file 4: Table S1. Explanation of how 92 studies provided data regarding 127 analyses. (DOC $36 \mathrm{~kb}$ )

\section{Abbreviations}

RNA, Ribonucleic acid; ncRNAs, Noncoding RNAs; LncRNAs, Long noncoding RNAs; LincRNA, large intergenic non-coding RNAs; T-UCRs, transcribed ultraconserved regions; miR, microRNA; HOTAIR (HOmeobox (HOX) Transcript Antlsense RNA); PRC2, Polycomb Repressive Complex 2; HR, Hazard Ratio; Cl, Confidence Interval; IQR, Interquartile range; DSS, Disease-specific survival; MFS, Metastasis-free survival, OS, Overall/cumulative survival; PFS, Progression/ event/disease-free survival; RFS, Recurrence-free survival; O, Number of observed events; E, Number of expected events; PCR, Polymerase chain reaction; qPCR, Quantitative PCR; qRT-PCR, Quantitative real-time PCR; RT-qPCR, real-time quantitative PCR; ISH, in situ hybridization; LNM, Lymph node metastasis; LVM, Lymphovascular metastasis

\section{Acknowledgements}

None.

Funding

No sources of funding to declare.

\section{Availability of data and materials}

The complete database upon which this review article has been constructed can be freely accessed here: https://goo.gl/EjCDAp. The size of this database does not permit its publication as an additional supporting file.

\section{Authors' contributions}

SS: study design, acquisition, analysis and interpretation of data, manuscript drafting; AK: study design, acquisition of data; final approval of the manuscript; JPA: study conception and design, data interpretation, drafting and critical appraisal of manuscript. All authors have given final approval to this version of the manuscript to be published.

\section{Authors' information}

As previously declared.

\section{Competing interests}

The authors declare that they have no competing interests.

\section{Consent for publication}

Not applicable.

Ethics approval and consent to participate

Not applicable.

\section{Author details}

${ }^{1}$ St. John's Hospital, Livingston EH54 6PP, UK. ${ }^{2}$ College of Medicine and Veterinary Medicine, University of Edinburgh, Edinburgh, UK. ${ }^{3}$ University Hospital of North Durham, North Rd, Durham DH1 5TW, UK. ${ }^{4}$ Stanford Prevention Research Center, Department of Medicine, Stanford University School of Medicine Stanford, Stanford, CA 94305, USA. ${ }^{5}$ Department of Health Research and Policy, Stanford University School of Medicine, Stanford, CA 94305, USA. ${ }^{6}$ Department of Statistics, Stanford University School of Humanities and Sciences, Stanford, CA 94305, USA. ${ }^{7}$ Meta-Research Innovation Center at Stanford (METRICS), Stanford University, 1265 Welch Rd, MSOB X306, Stanford, CA 94305, USA.

Received: 18 February 2016 Accepted: 14 June 2016

Published online: 28 June 2016

References

1. Alexander RP, Fang G, Rozowsky J, Snyder M, Gerstein MB. Annotating non-coding regions of the genome. Nat Rev Genet. 2010;11:559-71.

2. Esteller M. Non-coding RNAs in human disease. Nat Rev Genet Nat Publish Group. 2011;12:861-74.

3. Djebali S, Davis CA, Merkel A, Dobin A, Lassmann T, Mortazavi A, et al. Landscape of transcription in human cells. Nature. 2012;489:101-8.

4. Kornienko AE, Guenzl PM, Barlow DP, Pauler FM. Gene regulation by the act of long non-coding RNA transcription. BMC Biol BioMed Central. 2013;11:1. 
5. Nair VS, Maeda LS, loannidis JPA. Clinical outcome prediction by microRNAs in human cancer: a systematic review. J Natl Cancer Inst. 2012;104:528-40.

6. Mercer TR, Dinger ME, Mattick JS. Long non-coding RNAs: insights into functions. Nat Rev Genet Nat Publish Group. 2009;10:155-9.

7. Guttman M, Donaghey J, Carey BW, Garber M, Grenier JK, Munson G, et al. lincRNAs act in the circuitry controlling pluripotency and differentiation. Nature. 2011;477:295-300.

8. Ponting CP, Oliver PL, Reik W. Evolution and functions of long noncoding RNAs. Cell. 2009;136:629-41.

9. Malek E, Jagannathan S, Driscoll JJ. Correlation of long non-coding RNA expression with metastasis, drug resistance and clinical outcome in cancer Oncotarget. 2014;5:8027-38.

10. Iyer MK, Niknafs YS, Malik R, Singhal U, Sahu A, Hosono Y, et al. The landscape of long noncoding RNAs in the human transcriptome. Nat Genet Nat Publish Group. 2015;47:199-208.

11. Lee JT. Epigenetic regulation by long noncoding RNAs. Science. 2012;338:1435-9.

12. Rinn JL, Chang HY. Genome regulation by long noncoding RNAs. Annu Rev Biochem. 2012;81:145-66.

13. Khalil AM, Guttman M, Huarte M, Garber M, Raj A, Rivea Morales D, et al. Many human large intergenic noncoding RNAs associate with chromatinmodifying complexes and affect gene expression. Proc Natl Acad Sci U S A. 2009;106:11667-72.

14. Koziol MJ, Rinn JL. RNA traffic control of chromatin complexes. Curr Opin Genet Dev. 2010;20:142-8.

15. Vance KW, Ponting CP. Transcriptional regulatory functions of nuclear long noncoding RNAs. Trends Genet. 2014;30(8):348-355.

16. Penny GD, Kay GF, Sheardown SA, Rastan S, Brockdorff N. Requirement for Xist in X chromosome inactivation. Nature. 1996:379:131-7.

17. Wutz A, Rasmussen TP, Jaenisch R. Chromosomal silencing and localization are mediated by different domains of Xist RNA. Nat Genet Nat Publish Group. 2002;30:167-74.

18. Wutz A, Gribnau J. X inactivation Xplained. Curr Opin Genet Dev. 2007; 17:387-93

19. Forne T, Oswald J, Dean W, Saam JR, Bailleul B, Dandolo L, et al. Loss of the maternal $\mathrm{H} 19$ gene induces changes in Igf2 methylation in both cis and trans. PNAS Nation Acad Sci. 1997:94:10243-8.

20. Gabory A, Ripoche M-A, Le Digarcher A, Watrin F, Ziyyat A, Forné T, et al. $\mathrm{H} 19$ acts as a trans regulator of the imprinted gene network controlling growth in mice. Dev Company Biol Ltd. 2009;136:3413-21.

21. Calin GA, Liu C-G, Ferracin M, Hyslop T, Spizzo R, Sevignani C, et al. Ultraconserved regions encoding ncRNAs are altered in human leukemias and carcinomas. Cancer Cell. 2007;12:215-29.

22. Spizzo R, Almeida MI, Colombatti A, Calin GA. Long non-coding RNAs and cancer: a new frontier of translational research? Oncogene. 2012:31:4577-87

23. Li X, Wu Z, Fu X, Han W. Long Noncoding RNAs: Insights from Biological Features and Functions to Diseases. Med Res Rev. 2013:33:517-53.

24. Gupta RA, Wang KC, Hung T, West RB, Sukumar S, Chang HY. Long non-coding RNA HOTAIR reprograms chromatin state to promote cancer metastasis. Nature. 2010;464:1071-6.

25. Wang J, Xu AM, Zhang JY, He XM, Pan YS, Cheng G, et al. Prognostic significance of long non-coding RNA MALAT-1 in various human carcinomas: a meta-analysis. Genet. Mol. Res. 2016;15(1).

26. Deng Q, Sun H, He B, Pan Y, Gao T, Chen J, et al. Prognostic Value of Long Non-Coding RNA HOTAIR in Various Cancers. PLOS ONE Public Library Sci. 2014;9:e110059.

27. Serghiou S, Patel CJ, Tan YY, Koay P, loannidis JPA. Field-wide meta-analyses of observational associations can map selective availability of risk factors and the impact of model specifications. J Clin Epidemiol. 2016;71:58-67

28. Liberati A, Altman DG, Tetzlaff J, Mulrow C, Gøtzsche PC, loannidis JPA, et al. The PRISMA statement for reporting systematic reviews and meta-analyses of studies that evaluate health care interventions: explanation and elaboration. PLoS Med. Public Library of Science; 2009;6(7):e1000100.

29. Parmar MK, Torri V, Stewart L. Extracting summary statistics to perform meta-analyses of the published literature for survival endpoints. Stat Med. 1998;17:2815-34

30. Altman DG. Systematic reviews of evaluations of prognostic variables. BMJ British Med J Publish Group. 2001;323:224-8.
31. Haynes RB, McKibbon KA, Wilczynski NL, Walter SD, Werre SR, Team H. Optimal search strategies for retrieving scientifically strong studies of treatment from Medline: analytical survey. BMJ. 2005;330:1179.

32. R Development Core Team. R: A Language and Environment for Statistical Computing [Internet]. Vienna, Austria. Available from: http://www.R-project.org.

33. Lu L, Zhu G, Zhang C, Deng Q, Katsaros D, Mayne ST, et al. Association of large noncoding RNA HOTAIR expression and its downstream intergenic CpG island methylation with survival in breast cancer. Breast Cancer Res Treat Springer US. 2012;136:875-83.

34. Moons KGM, De Groot JAH, Bouwmeester W, Vergouwe Y, Mallett S, Altman DG, et al. Critical appraisal and data extraction for systematic reviews of prediction modelling studies: the CHARMS checklist. Plos Med Public Library Sci. 2014;11:e1001744

35. Xu Z-Y, Yu Q-M, Du Y-A, Yang L-T, Dong R-Z, Huang L, et al. Knockdown of long non-coding RNA HOTAIR suppresses tumor invasion and reverses epithelial-mesenchymal transition in gastric cancer. Int J Biol Sci. 2013;9:587-97.

36. Wu Z-H, Wang X-L, Tang H-M, Jiang T, Chen J, Lu S, et al. Long non-coding RNA HOTAIR is a powerful predictor of metastasis and poor prognosis and is associated with epithelial-mesenchymal transition in colon cancer. Oncol Rep Spandidos Publ. 2014;32:395-402.

37. Takahashi Y, Sawada G, Kurashige J, Uchi R, Matsumura T, Ueo H, et al. Amplification of PVT-1 is involved in poor prognosis via apoptosis inhibition in colorectal cancers. Br J Cancer. 2014;110:164-71.

38. Bouwmeester W, Zuithoff NPA, Mallett S, Geerlings MI, Vergouwe Y, Steyerberg EW, et al. Reporting and methods in clinical prediction research: a systematic review. Macleod MR, editor. Plos Med Public Library of Sci. 2012:9:1-12.

39. McShane LM, Altman DG, Sauerbrei W, Taube SE, Gion M, Clark GM. REporting recommendations for tumour MARKer prognostic studies (REMARK). Br J Cancer Nat Publish Group. 2005;93:387-91.

40. Henry NL, Hayes DF. Cancer biomarkers. Mol Oncol. 2012:6:140-6.

41. Higgins J, Thompson SG. Quantifying heterogeneity in a meta-analysis. Stat Med. 2002;21(11):1539-58.

42. Ioannidis JPA, Patsopoulos NA, Evangelou E. Uncertainty in heterogeneity estimates in meta-analyses. BMJ British Med J Publish Group. 2007:335:914-6.

43. Viechtbauer W. Conducting meta-analyses in $\mathrm{R}$ with the metafor package. J Statistical Software [Internet]. 2010;36:1-48. Available from: http://www. jstatsoft.org/v36/i03/.

44. Ioannidis JPA, Trikalinos TA. An exploratory test for an excess of significant findings. Clin Trials. 2007:4:245-53.

45. Ioannidis JPA. Clarifications on the application and interpretation of the test for excess significance and its extensions. J Math Psychol. 2013;57:184-7.

46. Simon R, Altman DG. Statistical aspects of prognostic factor studies in oncology. Br J Cancer Nat Publish Group. 1994;69:979-85.

47. Blettner M, Sauerbrei W, Schlehofer B, Scheuchenpflug T, Friedenreich C. Traditional reviews, meta-analyses and pooled analyses in epidemiology. Int J Epidemiol. 1999;28:1-9.

48. Kyzas PA, Loizou KT, loannidis JPA. Selective reporting biases in cancer prognostic factor studies. J Natl Cancer Inst Oxford Univ Press. 2005:97:1043-55.

49. Sideris M, Papagrigoriadis S. Molecular biomarkers and classification models in the evaluation of the prognosis of colorectal cancer. Anticancer Res. 2014;34:2061-8

50. Weigel MT, Dowsett M. Current and emerging biomarkers in breast cancer: prognosis and prediction. Endocr Relat Cancer BioScientifica. 2010;17:R245-62.

51. Altman DG, McShane LM, Sauerbrei W, Taube SE. Reporting Recommendations for Tumor Marker Prognostic Studies (REMARK): explanation and elaboration. Plos Med Public Library Sci. 2012;9:e1001216.

52. Kyzas PA, Denaxa-Kyza D, loannidis JPA. Almost all articles on cancer prognostic markers report statistically significant results. Eur J Cancer. 2007:43:2559-79.

53. Peat G, Riley RD, Croft P, Morley Kl, Kyzas PA, Moons KGM, et al. Improving the transparency of prognosis research: the role of reporting, data sharing, registration, and protocols. Plos Med Public Library of Sci. 2014;11:e1001671.

54. Moons KGM, Altman DG, Reitsma JB, loannidis JPA, Macaskill P, Steyerberg EW, et al. Transparent Reporting of a multivariable prediction model for Individual Prognosis or Diagnosis (TRIPOD): explanation and elaboration. Ann Intern Med Am College of Physicians. 2015:162:W1-W73.

55. Janssens ACJW, loannidis JPA, van Duijn CM, Little J, Khoury MJ, GRIPS Group. Strengthening the reporting of Genetic Rlsk Prediction Studies: the GRIPS Statement. Plos Med. 2011;8(3):e1000420. 
56. Altman DG, Vergouwe Y, Royston P, Moons KGM. Prognosis and prognostic research: validating a prognostic model. BMJ. 2009;338:b605.

57. Siontis GCM, Tzoulaki I, Castaldi PJ, loannidis JPA. External validation of new risk prediction models is infrequent and reveals worse prognostic discrimination. J Clin Epidemiol. 2015;68:25-34.

58. Ioannidis JPA. Why most discovered true associations are inflated. Epidemiology. 2008;19:640-8.

59. Sterne JA, Gavaghan D, Egger M. Publication and related bias in meta-analysis: power of statistical tests and prevalence in the literature. J Clin Epidemiol. 2000;53:1119-29.

60. IntHout J, loannidis JPA, Borm GF, Goeman JJ. Small studies are more heterogeneous than large ones: a meta-meta-analysis. J Clin Epidemiol. 2015;68:860-9

61. Ioannidis JPA, Panagiotou OA. Comparison of effect sizes associated with biomarkers reported in highly cited individual articles and in subsequent meta-analyses. JAMA Am Med Assoc. 2011;305:2200-10.

62. Pan Z, Trikalinos TA, Kavvoura FK, Lau J, loannidis JPA. Local literature bias in genetic epidemiology: an empirical evaluation of the Chinese literature. Plos Med Public Library Sci. 2005;2:e334.

63. Ioannidis JPA, Chang CQ, Lam TK, Schully SD, Khoury MJ. The geometric increase in meta-analyses from China in the genomic era. PLOS ONE Public Library Sci. 2013;8:e65602.

64. Hayes DF, Bast RC, Desch CE, Herbert Fritsche J, Kemeny NE, Jessup JM, et al. Tumor Marker Utility Grading System: a Framework to Evaluate Clinical Utility of Tumor Markers. J Natl Cancer Inst Oxford Univ Press. 1996:88:1456-66.

65. Harris L, Fritsche H, Mennel R, Norton L, Ravdin P, Taube S, et al. American Society of Clinical Oncology 2007 update of recommendations for the use of tumor markers in breast cancer. J Clin Oncol Am Soc Clin Oncol. 2007;25:5287-312.

66. Chi Y, Huang S, Yuan L, Liu M. Role of BC040587 as a predictor of poor outcome in breast cancer. Cancer Cell Int. 2014;14(1):123.

67. Liu PY, Erriquez D, Marshall GM, Tee AE, Polly P, Wong M, et al. Effects of a novel long noncoding RNA, InCUSMyCN, on N-Myc expression and neuroblastoma progression. J Natl Cancer Inst. 2014;106:113-3. dju.

\section{Submit your next manuscript to BioMed Central and we will help you at every step:}

- We accept pre-submission inquiries

- Our selector tool helps you to find the most relevant journal

- We provide round the clock customer support

- Convenient online submission

- Thorough peer review

- Inclusion in PubMed and all major indexing services

- Maximum visibility for your research

Submit your manuscript at www.biomedcentral.com/submit
Biomed Central 